\title{
Fetal hemorrhage and platelet dysfunction in SLP-76-deficient mice
}

\author{
James L. Clements, ${ }^{1}$ Jong Ran Lee, ${ }^{1}$ Barbara Gross, ${ }^{2}$ Baoli Yang, ${ }^{3}$ John D. Olson, ${ }^{4}$ \\ Alexander Sandra, ${ }^{5}$ Stephen P. Watson, ${ }^{2}$ Steven R. Lentz, ${ }^{6}$ and Gary A. Koretzky ${ }^{7}$ \\ ${ }^{1}$ Department of Internal Medicine, University of Iowa College of Medicine, Iowa City, Iowa 52242, USA \\ ${ }^{2}$ Department of Pharmacology, University of Oxford, Oxford OX1 3QT, United Kingdom \\ ${ }^{3}$ Department of Obstetrics and Gynecology, \\ ${ }^{4}$ Department of Pathology, and \\ ${ }^{5}$ Department of Anatomy and Cell Biology, University of Iowa College of Medicine, Iowa City, Iowa 52242, USA \\ ${ }^{6}$ Department of Internal Medicine, University of Iowa College of Medicine and Veterans Affairs Medical Center, \\ Iowa City, Iowa 52242, USA \\ ${ }^{7}$ Departments of Internal Medicine, Physiology and Biophysics, and the Interdisciplinary Immunology Program, University of Iowa \\ College of Medicine, Iowa City, Iowa 52242, USA
}

Address correspondence to: Gary A. Koretzky, Department of Internal Medicine, 540 EMRB, University of Iowa College of Medicine, Iowa City, Iowa 52242, USA. Phone: (319) 335-6844; Fax: (319) 335-6887; E-mail: gary-koretzky@uiowa.edu

James L. Clements and Jong Ran Lee contributed equally to this work.

Received for publication September 23, 1998, and accepted in revised form November 19, 1998.

\begin{abstract}
The adapter protein SLP-76 is expressed in T lymphocytes and hematopoietic cells of the myeloid lineage, and is known to be a substrate of the protein tyrosine kinases that are activated after ligation of the T-cell antigen receptor. Transient overexpression of SLP-76 in a T-cell line potentiates transcriptional activation after T-cell receptor ligation, while loss of SLP-76 expression abrogates several T-cell receptor-dependent signaling pathways. Mutant mice that lack SLP-76 manifest a severe block at an early stage of thymocyte development, implicating SLP-76 in signaling events that promote thymocyte maturation. While it is clear that SLP-76 plays a key role in development and activation of Tlymphocytes, relatively little is understood regarding its role in transducing signals initiated after receptor ligation in other hematopoietic cell types. In this report, we describe fetal hemorrhage and perinatal mortality in SLP-76-deficient mice. Although megakaryocyte and platelet development proceeds normally in the absence of SLP-76, collagen-induced platelet aggregation and granule release is markedly impaired. Furthermore, treatment of SLP-76-deficient platelets with collagen fails to elicit tyrosine phosphorylation of phospholipase C- $\gamma 2$ (PLC- $\gamma 2$ ), suggesting that SLP-76 functions upstream of PLC- $\gamma 2$ activation. These data provide one potential mechanism for the fetal hemorrhage observed in SLP-76-deficient mice and reveal that SLP-76 expression is required for optimal receptor-mediated signal transduction in platelets as well as T lymphocytes.
\end{abstract}

J. Clin. Invest. 103: 19-25 (1999)

\section{Introduction}

Adapter proteins play a critical role in the regulation of signal transduction events elicited after engagement of cell surface receptors. One such adapter protein is src homology 2 (SH2) domain-containing leukocyte protein of $76 \mathrm{kDa}$ (SLP-76), which was isolated initially from $\mathrm{T}$ cells as a novel substrate of the T-cell antigen receptor (TCR)-stimulated protein tyrosine kinases (PTK) (1). Examination of the primary structure and the initial biochemical characterization of SLP-76 indicated no evidence for enzymatic function of this protein, but revealed three domains capable of directing intermolecular interactions. SLP-76 contains several $\mathrm{NH}_{2}$-terminal tyrosine phosphorylation sites $(2,3)$, a central prolinerich region, and a $\mathrm{COOH}$-terminal SH2 domain (1). As predicted, all three domains of SLP-76 associate with other proteins in resting or activated $\mathrm{T}$ cells. These include Vav, which binds SLP-76 via the SH2 domain of Vav and phosphorylated tyrosines of SLP-76 $(4,5)$; growth factor binding protein 2 (Grb2), which associates with the proline-rich region of SLP-76 (6); and two phos- phoproteins (SLP-76-associated phosphoprotein of 130 $\mathrm{kDa}$ [SLAP-130] and an unidentified $62-\mathrm{kDa}$ protein), which bind the SH2 domain of SLP-76 $(6,7)$.

Interest in SLP-76 increased when it was found that overexpression of this adapter protein in transformed Tcell lines markedly augments TCR-stimulated transcription of the interleukin-2 gene (6). It appears that it is the adapter function of SLP-76 that is critical for its ability to modulate TCR-induced signals, because mutation of any of the three SLP-76 domains abrogates the enhanced signaling (8). A role for SLP-76 in signaling via the TCR in mature peripheral $\mathrm{T}$ cells as well as in signaling via the preTCR in developing thymocytes was suggested by the observation that expression of SLP-76 is regulated during T-cell development and activation (9). In support of this notion, a mutant variant of the Jurkat T-cell line that has lost SLP-76 expression exhibits a severe block in TCR signals as assessed by induced changes in intracellular free calcium and activation of the extracellular signal-regulated kinase (ERK) pathway (10). Furthermore, mice made deficient in SLP-76 expression by homologous recombi- 
nation manifest a complete block in T-cell development at an early stage of maturation presumably resulting from a failure of signal transduction via the pre-TCR $(11,12)$.

In addition to defects in pre-TCR signaling in SLP76-deficient mice, there are less than the expected numbers of SLP-76-deficient mice in weaned litters (12), suggesting a selective loss of SLP-76-deficient mice in utero or perinatally. Indeed, SLP-76-deficient are reported to selectively perish within the first week after birth (12). Here we confirm that SLP-76-deficient fetuses are present at the expected frequency in utero and appear morphologically normal as late as day 19 of gestation with the exception of striking subcutaneous hemorrhage. As a potential explanation for the bleeding disorder, we determined the ability of platelets to develop and function in the absence of SLP-76. We found that while SLP-76 is expressed in normal murine platelets, loss of SLP-76 expression results in only mild thrombocytopenia in the deficient mice. However, platelet function in response to collagen, an agonist that results in SLP-76 phosphorylation in normal mice, is lost completely in the SLP76-deficient strain. In contrast, platelets from the SLP76-deficient mice respond normally to thrombin, an agonist that does not induce significant phosphorylation of SLP-76 in platelets from wild-type animals. We provide evidence placing SLP-76 distal to Syk kinase activation and proximal to phospholipase C- $\gamma 2$ (PLC- $\gamma 2$ ) tyrosine phosphorylation in a collagen-dependent platelet signaling pathway. These studies underscore the importance of SLP-76 and adapter proteins in general in regulating cell surface receptor-dependent signaling events.

\section{Methods}

Mice. The generation of a SLP-76-deficient mouse strain has been described previously (11). All mice were housed under pathogenfree conditions at the University of Iowa Animal Care Facility and used in accordance with National Institutes of Health guidelines.

Preparation of tissue sections and samples for microscopic analysis. Fetuses were harvested at various stages of gestation, and a small tissue sample was obtained for the isolation of genomic DNA and genotyping by PCR. Fetuses were then fixed in 10\% formalin, embedded in paraffin, sectioned, and then stained with hematoxylin and eosin. Adult mice were sacrificed, and slides of peripheral blood smears or bone marrow were immediately prepared and stained with Wright-Giemsa stain. Sternum and humerus were removed, fixed in the presence of mercuric chloride, and then decalcified in 5\% nitric acid. Paraffin-embedded sections were then prepared and stained with hematoxylin and eosin. For electron microscopic analysis, whole blood was isolated and the platelet layer was fixed with glutaraldehyde after centrifugation. The platelet layer was removed, sectioned, and analyzed by transmission electron microscopy.

\section{Table 1}

Frequency of SLP-76 +/+, +/-, and -/- mice in utero and after weaning

\begin{tabular}{lccc}
\hline & $+/+$ & $+/-$ & $-/-$ \\
After weaning $(n=226)$ & $69(30.5 \%)$ & $138(61.1 \%)$ & $19(8.4 \%)$ \\
In utero (E12-E19; $n=72)$ & $19(26.4 \%)$ & $37(51.4 \%)$ & $16(22.2 \%)$
\end{tabular}

Genotypes were determined by PCR analysis using genomic DNA obtained from tail clips isolated at weaning (2-3 weeks after birth) or from tissue samples isolated from fetuses at the indicated days of gestation.

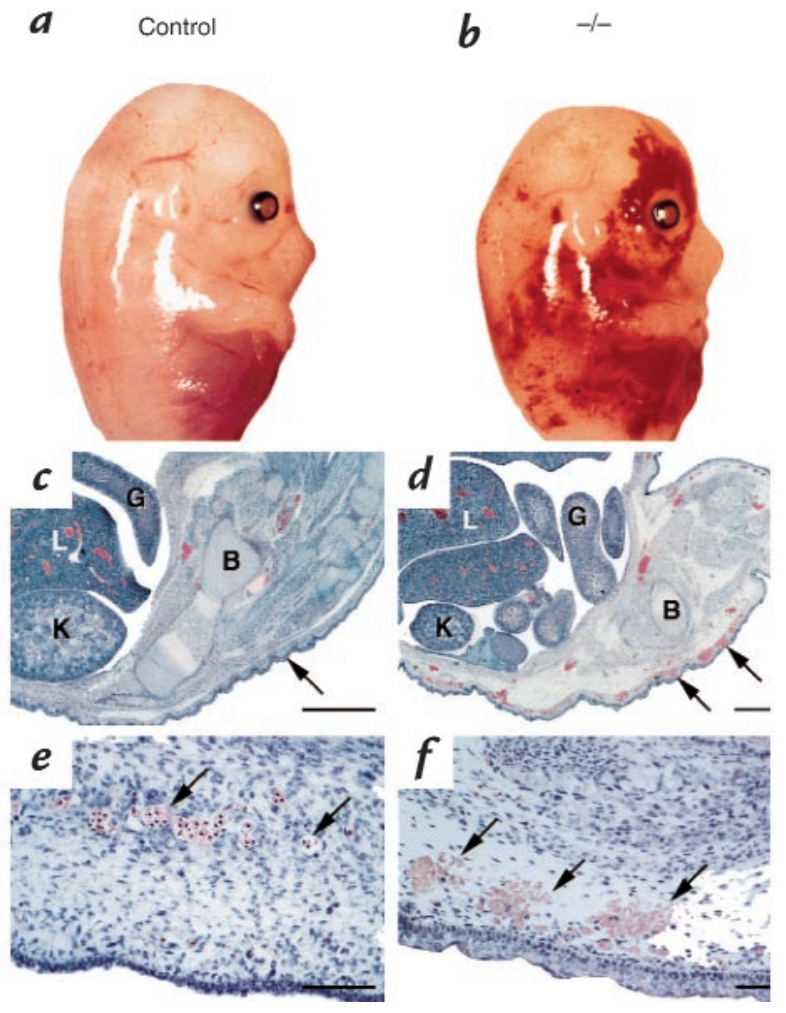

Figure 1

SLP-76-deficient mice manifest diffuse, subcutaneous hemorrhage and edema. SLP- $76^{+/-}$mice were mated and day of gestation was calculated based on the presence of a vaginal plug. At approximately day 14 ( $\boldsymbol{a}$ and $\boldsymbol{b})$ or day $18(\boldsymbol{c}-\boldsymbol{f})$ of gestation, the mother was sacrificed and fetuses were isolated. Genotypes were determined by PCR analysis using genomic DNA isolated from a small tissue sample as template. ( $a$ and $b$ ) Gross morphological appearance of littermate control (a) or SLP-76 ${ }^{-/}$(b) E14 fetuses. $(c-f)$ Histological appearance of SLP-76 $6^{+-}(c$ and $e)$ and SLP-76 $6^{-/-}$ ( $d$ and $f$ ) fetal sections at day 18 of gestation. ( $c$ and $d$ ) Caudal view of embryos. Note close association of epithelium with underlying connective tissue (arrow) in the SLP-76 $76^{+-}$fetus and edema and subcutaneous bleeding in the SLP-76-/- fetus. $B$, bone; $G$, gut; $K$, kidney; $L$, liver. Bar represents $1000 \mu \mathrm{M}$. ( $e$ and $f$ ) High-power view of subcutaneous region. Note intact endothelium and intraluminal nucleated red blood cells (arrows) in SLP-76 ${ }^{+/-}$embryo compared with attenuated endothelium, extravasated blood (arrow), and subcutaneous edema in the SLP-76-1embryo. Bar represents $200 \mu \mathrm{M}$.

Immunoprecipitation and Western blotting. Whole blood was collected by cardiac puncture into 0.1 vol of $3.8 \%$ sodium citrate. Platelet-rich plasma (PRP) was isolated after low-speed centrifugation (200 $\mathrm{g}$ for $10 \mathrm{~min}$ ), and platelet number was determined by automated analysis (Technicon H3; Bayer Inc., White Plains, New York, USA) PRP was centrifuged at $1,000 \mathrm{~g}$ for 5 min, and the platelet pellet was resuspended in Roswell Park Memorial Institute medium (RPMI-1640). Approximately 250 $\times 10^{6}$ platelets per condition were rested for $30 \mathrm{~min}$ and then left untreated or stimulated in the presence of native type I equine collagen $(100 \mu \mathrm{g} / \mathrm{ml})$, human thrombin $(100 \mathrm{nM})$, or pervanadate. Platelet samples were then lysed in 1\% NP-40. For analysis of SLP-76 expression and phosphorylation, lysates were subjected to immunoprecipitation with sheep polyclonal antimurine SLP-76. Immunoprecipitates were then washed, resolved by SDS-PAGE, transferred to nitrocellulose, and then blotted with an anti-phosphotyrosine-specific antibody (4G10; Upstate Biotechnology Inc., Lake Placid, New York, USA). After incubation in the presence of horseradish peroxidase-conjugated goat 

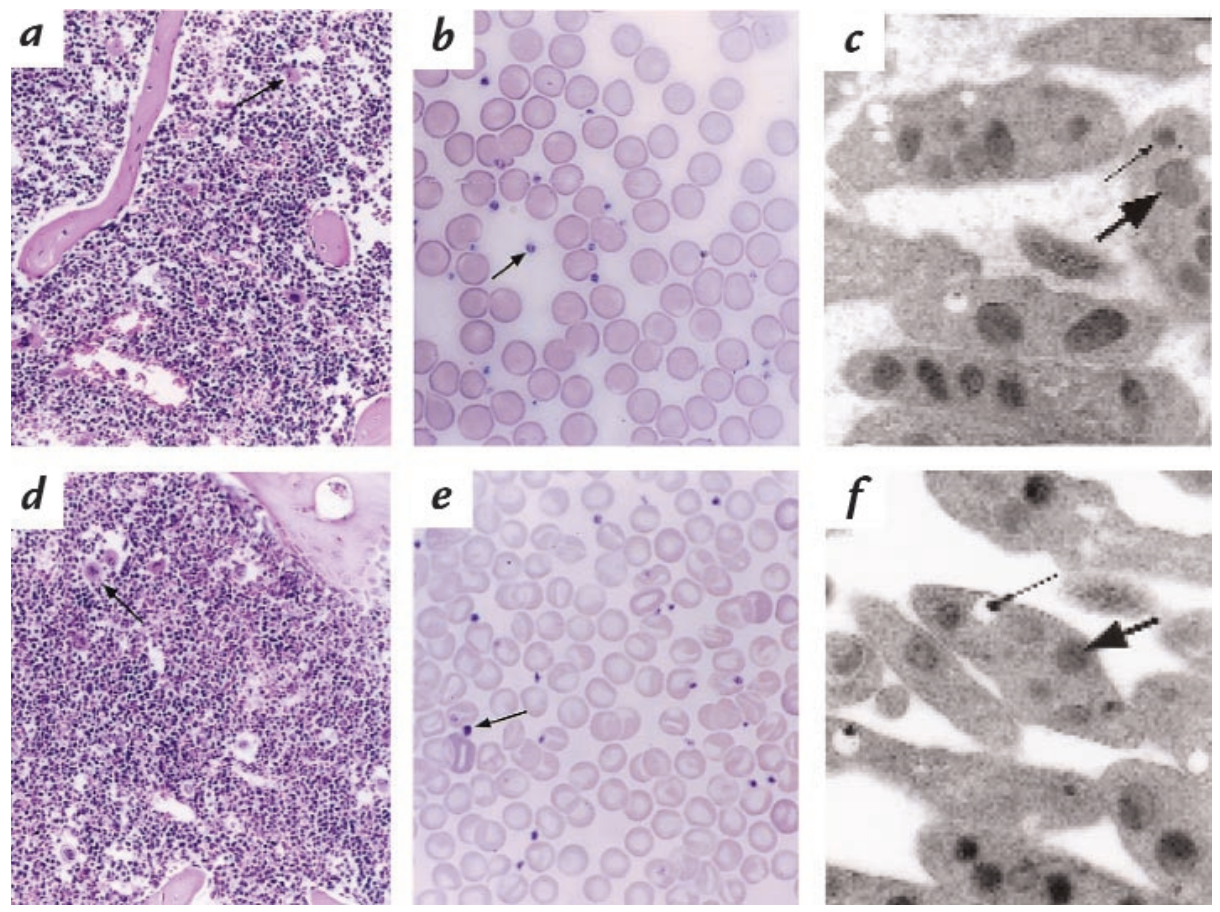

\section{Figure 2}

Platelets and megakaryocytes develop normally and exhibit normal morphology in the absence of SLP-76. The humerus and peripheral blood were isolated from SLP-76 $6^{+/+}(\boldsymbol{a}-\boldsymbol{c})$ or SLP-76 $6^{-/}(\boldsymbol{d}-\boldsymbol{f})$ mice. Bone marrow sections $(a$ and $d)$, whole blood smears $(b$ and $e)$, or glutaraldehyde-fixed platelet sections were generated as described in Methods. $(a$ and $d$ ) Hematoxylin and eosin-stained humeral bone marrow sections $(\times 100)$. Megakaryocytes are indicated with arrows. Note that the density of megakaryocytes in sections obtained from SLP-76 $6^{+/+}$and SLP-76-/- mice was similar (1.1-1.15 megakaryocytes per oil immersion field). ( $b$ and $e$ ) Wright-Giemsa staining of whole blood smears $(\times 250)$. Platelets are indicated with arrows. $(c$ and $f)$ Transmission electron microscopic analysis of glutaraldehyde-fixed platelets. Large arrows indicate $\alpha$ granules and small arrows denote dense granules.

anti-mouse IgG, bands were detected by enhanced chemiluminescence (ECL; Amersham Life Sciences Inc., Arlington Heights, Illinois, USA). Immunoprecipitation and immunoblot analysis of Syk and PLC- $\gamma 2$ was performed as described previously (19), with the exception that rabbit polyclonal anti-PLC- $\gamma 2$ (Santa Cruz Biotechnology Inc., Santa Cruz, California, USA) was used for immunoprecipitation and detection of PLC- $\gamma 2$.

Bleeding time. Adult mice were anesthetized with Avertin (10 $\mu \mathrm{g} / \mathrm{g}$ ), and a small standardized incision (1 $\mathrm{mm}$ long, $2 \mathrm{~mm}$ deep) was made $\sim 10-12 \mathrm{~mm}$ from the tip of the tail. Incisions were then immersed in $0.9 \%$ saline at room temperature, and bleeding time was defined as the time point at which all visible signs of bleeding from the incision had stopped.

Platelet aggregation and ATP release. Mice 8-12 weeks old were sacrificed by $\mathrm{CO}_{2}$ asphyxiation, and whole blood samples were immediately collected by cardiac puncture as described above. Blood cell counts and hematocrit were determined by automated analysis. Accuracy of automated quantification of platelets was confirmed by manual counting of erythrocytes and platelets in blood smears. Whole blood was diluted with saline to achieve a platelet concentration of $\sim 200 \times 10^{6}$ cells $/ \mathrm{ml}$ before analysis. Aggregation as measured by change in impedance, and ATP release from dense granules (luciferase-ATP-dependent luminescence) was monitored continuously via lumiaggregometry (Chrono-Log, Havertown, Pennsylvania, USA) after addition of bovine thrombin $(0.5 \mathrm{U} / \mathrm{ml})$ or native type I equine collagen $(20$ $\mu \mathrm{g} / \mathrm{ml}$ ) to the reaction cuvette.

\section{Results}

Mice made deficient for SLP-76 by homologous recombination appear healthy, but represent only $8.4 \%$ of offspring $(n=226)$ obtained from heterozygous matings
(Table 1). In contrast, the frequency of SLP-76-/- fetuses isolated between embryonic day 12 (E12) and embryonic day 19 (E19) of gestation is closer to the expected 25\% $(22.2 \% ; n=72)$, suggesting that SLP-76-deficient mice are not selectively lost before late stages of gestation. Gross examination of SLP-76 ${ }^{-/-}$fetuses as early as E12 provides a potential explanation for the perinatal mortality of these mice. These fetuses exhibit diffuse subcutaneous hemorrhage that becomes more obvious at later stages of fetal development (Fig. 1, $a$ and $b$ ). Histo-

\section{Table 2}

Analysis of peripheral blood obtained from SLP-76-deficient mice and littermate controls

$\begin{array}{lll}+/+ & +/- & -1-\end{array}$

$\begin{array}{lccc}\text { WBC }(\times 1,000 / \mu l) & 4.3 \pm 0.4^{\mathrm{A}} & 7.3 \pm 1.3 & 7.3 \pm 1.2^{\mathrm{B}} \\ \operatorname{HGB}(\mathrm{g} / \mathrm{dl}) & 12.4 \pm 0.2 & 12.2 \pm 0.5 & 11.0 \pm 0.3^{\mathrm{B}} \\ \mathrm{HCT}(\%) & 39.3 \pm 1.4 & 39.5 \pm 1.1 & 36.3 \pm 1.3 \\ \operatorname{RDW}(\%) & 11.2 \pm 0.5 & 11.8 \pm 0.4 & 13.5 \pm 0.5^{\mathrm{B}} \\ \operatorname{PLT}(\times 1,000 / \mu \mathrm{l}) & 951 \pm 83 & 950 \pm 72 & 610 \pm 79^{\mathrm{B}}\end{array}$

The hematologic profile of peripheral blood was determined by automated analysis and confirmed by manual quantification where possible. Statistical analysis was carried out by nonparametric comparisons (+/+ vs. +/-, +/- vs. -/-) using the Wilcoxon Mann-Whitney test. All tests were two-sided and all analyses were completed using SAS.

AData are presented as mean $\pm \operatorname{SEM}(n=4)$.

${ }^{\mathrm{B}} \mathrm{P}<0.05$ vs. SLP-76 $6^{+/+}$

$W B C$, white blood cells; $H G B$, hemoglobin; $H C T$, hematocrit; RDW, red blood cell distribution width; PLT, platelets. 


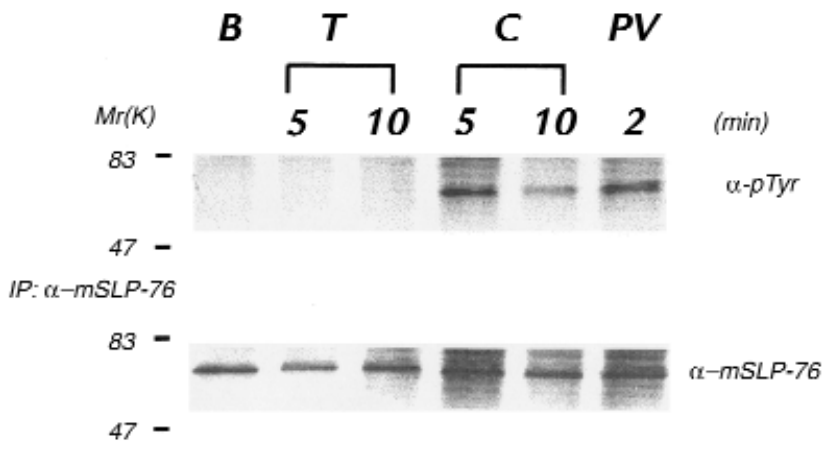

Figure 3

SLP-76 is tyrosine-phosphorylated in response to collagen. Platelets were isolated from normal mice and left untreated (basal, $B$ ) or incubated with thrombin $(T)$, collagen $(C)$, or pervanadate $(P V)$ for the times indicated. Platelets were then lysed and subjected to immunoprecipitation with a murine SLP-76-specific antibody. Immunoprecipitates were washed, resolved by SDS-PAGE, transferred to nitrocellulose, and then immunoblotted with the phosphotyrosine-specific antibody 4G10 $(\alpha-$ pTyr). The immunoblot shown in the top panel was stripped and reblotted with an SLP-76-specific antibody (SLP-76) to demonstrate equal amounts of immunoprecipitated SLP-76 in each lane. Identical results were obtained in a separate experiment.

logical examination of SLP-76-/- fetuses at E18 reveals normal organogenesis and morphology (compare Fig. $1, c$ and $d$ ). However, prominent extravasation of red blood cells and subcutaneous edema are apparent (compare Fig. 1, $e$ and $f$ ), consistent with the gross appearance of the SLP-76-/- fetuses. Thus, it appears that SLP76-deficient fetuses develop normally through late stages of gestation but exhibit an apparent bleeding disorder that may contribute to the premature death of these mice shortly after birth (12).

Examination of SLP-76/- mice at 8-12 weeks of age reveals spontaneous intraperitoneal hemorrhage, which suggests that the bleeding diathesis observed in utero is maintained into adulthood. To begin to address the nature of the bleeding disorder, we determined the hematological profile of peripheral blood obtained from SLP-76 +/+,+/-, and -/- mice. Compared with littermate controls, SLP-76 ${ }^{-/}$mice exhibit mild anemia, mild thrombocytopenia, and increased red cell distribution width (RDW) (Table 2). Although SLP-76 ${ }^{-/-}$mice have reduced peripheral platelet counts, bone marrow sections from SLP-76 ${ }^{-/-}$mice and littermate controls reveal comparable numbers of megakaryocytes that display normal morphology (compare Fig. 2, $a$ and $d$ ). Light or transmission electron microscopic analysis of whole blood smears (Fig. 2, $b$ and $e$ ) or purified platelets (Fig. 2, $c$ and $f$ ) reveal no major gross or structural morphological differences between platelets isolated from control and SLP-76 $\%$ mice. Thus, in the absence of SLP-76, megakaryocyte development and platelet production appear to proceed normally. These findings, coupled with the observation that more severe thrombocytopenia in other mutant mouse strains does not lead to significant bleeding (13-15), support the notion that hemorrhage in fetal and adult SLP-76-/- mice is not caused solely by decreased platelet number.
We next determined the prothrombin time (PT) and partial thromboplastin time (PTT) using plasma isolated from control or SLP-76-/- mice as a means to assess the extrinsic and intrinsic coagulation pathways, respectively. These studies revealed no difference between SLP-76 $+/+,+/-$, or $-/-$ mice (data not shown), suggesting that the observed fetal hemorrhage in SLP-76 $6^{-/}$mice is not due to defects in these coagulation pathways. We therefore focused our attention on a potential qualitative defect in platelet function as a possible cause for the bleeding disorder observed in SLP-76-/- mice. SLP-76 is expressed in human platelets and is tyrosine-phosphorylated after ligation of FcyRIIA (16). SLP-76 is also expressed in freshly isolated murine platelets, and it undergoes rapid tyrosine phosphorylation after treatment of platelets with collagen, but not thrombin (Fig. 3), suggesting that SLP-76 is a substrate of tyrosine kinases activated after collagen receptor ligation. Platelets isolated from adult SLP-76 $+/+,+/-$, and -/- mice aggregate and release ATP from granules comparably in response to thrombin (Fig. 4, a and $b$ ). However, SLP-76-/- platelets manifest a marked impairment in these responses after exposure to collagen (Fig. 4, $a$ and $b$ ), demonstrating that collagen-induced signaling is impaired in the absence of SLP-76. It should be noted that the enhanced responsiveness of platelets isolated from SLP-76 ${ }^{+/-}$mice observed in Fig. $4 a$ was not seen in other experiments. Despite the collagen-specific platelet defect manifested by SLP-76 ${ }^{-/-}$mice, tail bleeding times obtained from SLP-76 ${ }^{-/-}$mice $(2.68 \pm 0.94$ minutes $)$ did not differ from those obtained from SLP-76 ${ }^{+/+}(2.62$ $\pm 1.38)$ or SLP-76 $6^{+/-}(3.12 \pm 1.22)$ control mice. These data imply that primary hemostasis as determined by this method is preserved in the absence of SLP-76 and collagen-specific platelet function.

Treatment of platelets with collagen has been demonstrated to induce the tyrosine phosphorylation of the Syk tyrosine kinase and phospholipase PLC- $\gamma 2(17,18)$. The required nature of these events for platelet function has been demonstrated by studies using mutant mouse strains which lack Syk or the FcR $\gamma$ chain (19), a membrane-associated protein that is thought to recruit Syk in a collagen-dependent manner (20). To define more precisely the impact of lost SLP-76 expression on collagendependent signaling pathways, we assayed for inducible tyrosine phosphorylation of Syk and PLC- $\gamma 2$ after collagen exposure. While exposure to collagen effectively induces tyrosine phosphorylation of Syk in platelets isolated from SLP-76 +/+, +/-, and -/- mice, the collagendependent phosphorylation of PLC- $\gamma 2$ is lost in the absence of SLP-76 (Fig. 5). These data suggest that SLP-76 functions to couple Syk activation with PLC- $\gamma 2$ phosphorylation, and they provide a potential biochemical explanation for the functional defect observed in SLP-76-deficient platelets after exposure to collagen.

\section{Discussion}

SLP-76-deficient embryos appear to develop normally with the exception of subcutaneous hemorrhage. While the expected number of SLP-76/- embryos derived from heterozygous matings are present in utero, there are fewer than the expected number of these mice in weaned litters. Consistent with this observation, Pivniouk et al. (12) 

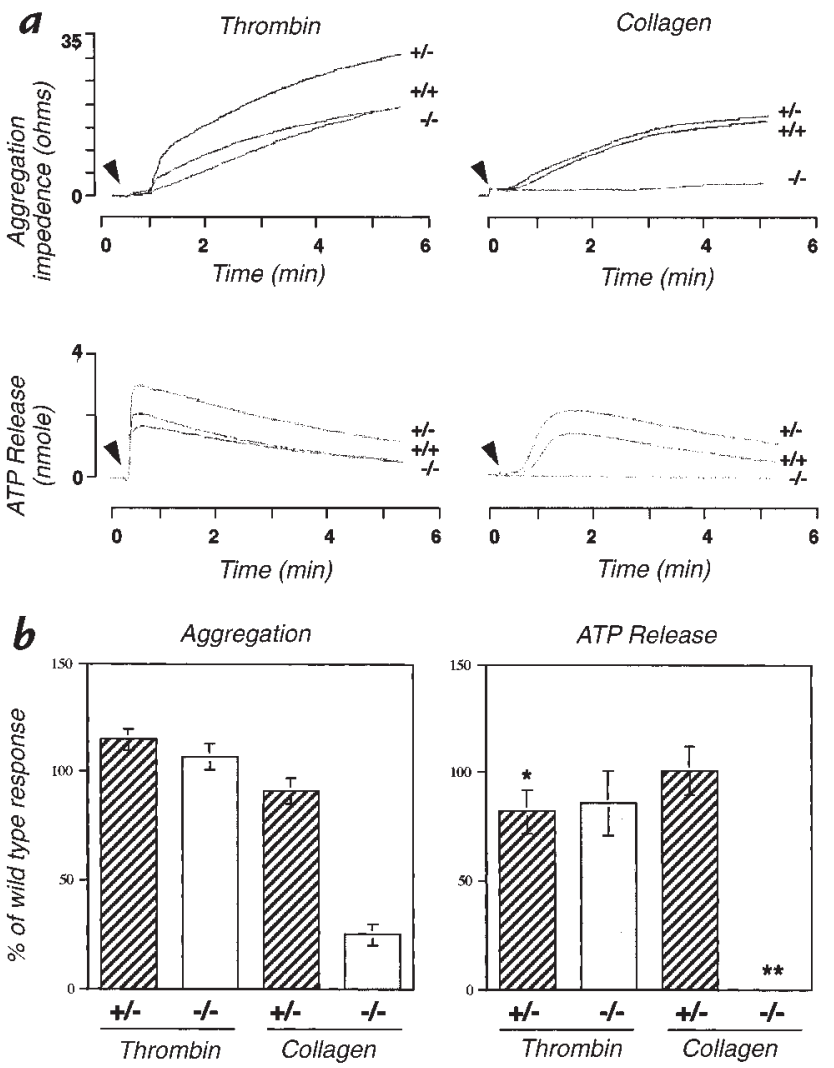

Figure 4

Platelet aggregation and ATP release in response to thrombin or collagen. Whole blood was isolated from SLP-76 +/+, +/-, or -/- mice, and platelet number was normalized before analysis. (a) Aggregation and ATP release tracings from one representative experiment. Arrowheads indicate the point at which the indicated agonist was added. (b) Change in impedence (Aggregation) or ATP concentration (ATP release) was determined for each sample at the same time point after addition of thrombin or collagen by measuring the amplitude of the response. The timepoint at which the response was measured correlated with the maximal response. The values obtained for the $+/-$ and -/- samples were normalized by calculating the percent of the $+/+$ response. The SEM for each group is shown. *These data were obtained from four independent experiments. In all other cases, data was obtained from five independent experiments. ${ }^{*}$ In all experiments, ATP release from SLP-76-/- platelets after exposure to collagen was not detectable.

have reported that SLP-76-deficient pups selectively perish within the first week after birth . Diffuse intraperitoneal hemorrhage is also observed in SLP-76-deficient mice that reach adulthood (our unpublished observations, and ref. 12). The hemorrhage observed in fetal and adult SLP-76-deficient mice prompted us to analyze blood samples for soluble and cellular components that mediate hemostasis. Both the intrinsic and extrinsic coagulation pathways are intact in SLP-76-deficient mice. Although SLP-76-deficient mice manifest mild thrombocytopenia, megakaryocytes, and platelets develop with apparently normal structural features. However, platelets isolated from SLP-76-deficient mice manifest an impaired ability to aggregate or undergo granule release in response to collagen while maintaining responsiveness to thrombin. In the absence of SLP-76, PLC- $\gamma 2$ is not tyrosine-phosphorylated after collagen exposure, despite the finding that inducible Syk phosphorylation is intact. Given the observation that SLP-76 is a substrate of tyrosine kinases activated after collagen receptor ligation, these data suggest that SLP-76 functions to couple proximal activation of tyrosine kinases with PLC- $\gamma 2$ phosphorylation in platelets.

Major platelet surface receptors for collagen include the $\alpha_{2} \beta_{1}$ integrin (glycoprotein Ia/IIa) and glycoprotein VI (21-24). While the $\alpha_{2} \beta_{1}$ integrin is required for the initial recruitment and adherence of the platelet to the collagen surface, glycoprotein VI has emerged as one of the primary candidates for initiating intracellular signaling after collagen binding $(25,26)$. Interestingly, several parallels have been identified between collagen-receptor and immune receptor signaling (27). Collagen-receptor engagement in platelets and TCR ligation in T lymphocytes both have been demonstrated to activate Syk family tyrosine kinases $(28,29)$. These kinases are recruited to tyrosine-phosphorylated immunoreceptor tyrosine-based activation motifs (ITAMs) present in the cytoplasmic chains of many immune receptor complexes, including the glycoprotein $\mathrm{VI}$-associated FcR $\gamma$ chain $(25,30-32)$. In contrast, thrombin receptors are demonstrated to signal predominantly via activation of heterotrimeric GTP-binding proteins (33, 34). Loss of FcR $\gamma$-chain expression results in markedly reduced tyrosine phosphorylation of Syk and PLC- $\gamma 2$ after exposure of platelets to collagen, suggesting that both of these enzymes function distal to the FcR $\gamma$ chain (19). While loss of Syk expression does not effect tyrosine phosphorylation of the FcR $\gamma$ chain or thrombin-induced aggregation, the inducible tyrosine phosphorylation of PLC- $\gamma 2$ in response to collagen is lost in Syk-deficient platelets, suggesting that Syk functions upstream of PLC$\gamma 2$ in these signaling pathways. These data have led to the description of a collagen-induced signaling cascade involving tyrosine phosphorylation of the FcR $\gamma$ chain, recruitment and activation of Syk, and the subsequent tyrosine phosphorylation and activation of PLC- $\gamma 2$ (19).

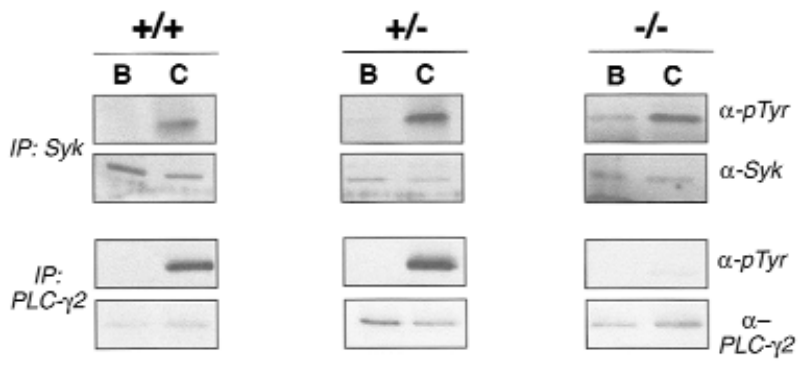

\section{Figure 5}

Collagen induces tyrosine phosphorylation of Syk but not PLC- $\gamma 2$ in SLP76-deficient platelets. Platelets were isolated from SLP-76 +/+, +/-, or $-/-$ mice and left resting (basal, $B$ ) or stimulated with collagen $(C)$ for $90 \mathrm{~s}$. Platelets were then lysed and subjected to immunoprecipitation with Syk-specific (top panels) or PLC- $\gamma 2$-specific (bottom panels) antibodies. Immunoprecipitates were washed, resolved by SDS-PAGE, and then transferred to nitrocellulose. Filters were then immunoblotted with phosphotyrosine-specific antibody $(\alpha-p$ Tyr $)$. Filters were then stripped and reblotted with Syk- or PLC- $\gamma 2$-specific antibodies to demonstrate equivalent sample loading. 
Loss of either FcR $\gamma$ chain or Syk markedly impairs platelet responses to collagen, emphasizing the biological importance of this signaling pathway (19).

Our data demonstrating that loss of SLP-76 expression abrogate the ability of platelets to aggregate normally or undergo granule release in response to collagen that SLP76 also plays an important role in collagen-receptor-initiated signaling. Given the structure of SLP-76, its required role in transducing pre-TCR and TCR-dependent signals $(10-12)$, and the observation that SLP-76 is a substrate for the Syk family tyrosine kinase ZAP-70 in T lymphocytes $(3,35)$, it seems likely that SLP-76 serves to translate Syk activation into more distal signaling pathways in platelets after exposure to collagen. In support of this notion, treatment of SLP-76-deficient platelets with collagen fails to elicit the inducible phosphorylation of PLC- $\gamma 2$. Furthermore, intracellular calcium release in SLP-76-deficient platelets is markedly impaired after collagen exposure (manuscript in preparation). The remarkably similar bleeding diathesis observed in both SLP-76-deficient and Syk-deficient $(36,37)$ fetuses further supports the notion that these proteins function in similar signaling pathways and suggests at least one potential consequence of impaired collagen-induced platelet activation. Several additional mutant mouse strains have been described that manifest defects in platelet development or function and display fetal hemorrhage. Severe thrombocytopenia and hemorrhage in the peritoneal cavity and gastrointestinal tract have been described in mice that lack the transcription factor NF-E2 (38). Defective platelet function and intra-abdominal bleeding have been reported in mice made deficient for G $\alpha q$ (39). Taken together, these studies suggest a potential relationship between platelet dysfunction and spontaneous hemorrhage.

While the impaired platelet response to collagen may contribute to the bleeding diathesis observed in SLP-76mice, the spontaneous hemorrhage observed in these mice may be attributable to other mechanisms, such as an endothelial cell defect or loss of other cell types that are normally required for the maintenance of vessel integrity. However, we detect no SLP-76 mRNA in primary human umbilical vein endothelial cells (data not shown), suggesting that SLP-76 is not normally expressed in the vascular endothelium. Loss of T lymphocytes alone in SLP-76-/- mice can not account for the bleeding diathesis, since other mutant mouse strains that fail to develop $\mathrm{T}$ lymphocytes have no obvious bleeding defects. It remains possible that other hematopoietic cell types that develop in the absence of SLP-76 may manifest functional defects or fail to produce soluble factors that influence vascular integrity. Megakaryocyte-specific reconstitution of SLP-76 in SLP-76 ${ }^{-/}$mice should facilitate the determination if platelet dysfunction is the primary cause of hemorrhage and premature death observed in the absence of SLP-76.

\section{Acknowledgments}

We thank Joyce Sanderson-Gillihan, Beverly Pennell, and Steve Eliason for expert technical assistance. We also thank Paul Reimann and Joel Carl for assistance with figure preparation. This work was supported in part by grants from the Arthritis Foundation (to J.L. Clements), the Wellcome Trust (to B.
Gross), the British Heart Foundation (to S.P. Watson), the National Institutes of Health and the University of Iowa Diabetes and Endocrinology Research Center (to G.A. Koretzky and S.R. Lentz), and the American Heart Association (to G.A. Koretzky and S.R. Lentz).

1. Jackman, J., et al. 1995. Molecular cloning of SLP-76, a $76 \mathrm{kDa}$ tyrosine phosphoprotein associated with Grb2 in T cells. J. Biol. Chem. 270:7029-7032.

2. Fang, N., Motto, D.G., Ross, S.E., and Koretzky, G.A. 1996. Tyrosines 113,128 , and 145 of SLP-76 are required for optimal augmentation of NFAT promoter activity. J. Immunol. 157:3769-3773.

3. Wardenburg, J.B., et al. 1996. Phosphorylation of SLP-76 by the ZAP-70 protein-tyrosine kinase is required for T-cell receptor function. J. Biol. Chem. 271:19641-19644

4. Wu, J., Motto, D.G., Koretzky, G.A., and Weiss, A. 1996. Vav and SLP-76 interact and functionally cooperate in IL-2 gene activation. Immunity. 4:593-602.

5. Tuosto, L., Michel, F., and Acuto, O. 1996. p95vav associates with tyrosine-phosphorylated SLP-76 in antigen- stimulated T cells. J. Exp. Med. 184:1161-1166

6. Motto, D.G., Ross, S.E., Wu, J., Hendricks-Taylor, L.R., and Koretzky, G.A. 1996. Implication of the GRB2-associated phosphoprotein SLP-76 in $\mathrm{T}$ cell receptor-mediated interleukin 2 production. J. Exp. Med. 183:1937-1943.

7. Musci, M., et al. 1997. Molecular cloning of SLAP-130, an SLP-76-associated substrate of the $\mathrm{T}$ cell antigen receptor-stimulated protein tyrosine kinases. J. Biol. Chem. 272:11674-11677.

8. Musci, M.A., Motto, D.G., Ross, S.E., Fang, N., and Koretzky, G.A. 1997. Three domains of SLP-76 are required for its optimal function in a T-cell line. J. Immunol. 159:1639-1647.

9. Clements, J.L., Ross-Barta, S.E., Tygrett, L.T., Waldschmidt, T.J., and Koretzky, G.A. 1998. SLP-76 expression is restricted to hematopoietic cells of monocyte, granulocyte, and T lymphocyte lineage and is regulated during $\mathrm{T}$ cell maturation and activation. J. Immunol. 161:3880-3881.

10. Yablonski, D., Kuhne, M.R., Kadlecek, T., and Weiss, A. 1998. Uncoupling of nonreceptor tyrosine kinases from PLC-gamma1 in an SLP-76 deficient T cell. Science. 281:413-416.

11. Clements, J.L., et al. 1998. Requirement for the leukocyte-specific adapter protein SLP-76 for normal T cell development. Science. 281:416-419.

12. Pivniouk, V., et al. 1998. Impaired viability and profound block in thymocyte development in mice lacking the adaptor protein SLP-76. Cell. 94:229-238.

13. Gurney, A.L., Carver-Moore, K., de Sauvage, F.J. and Moore, M.W. 1994. Thrombocytopenia in c-mpl-deficient mice. Science. 265:1445-1447.

14. de Sauvage, F.J., et al. 1996. Physiological regulation of early and late stages of megakaryocytopoiesis by thrombopoietin. J. Exp. Med. 183:651-656

15. Bunting, S., et al. 1997. Normal platelets and megakaryocytes are produced in vivo in the absence of thrombopoietin. Blood. 90:3423-3429.

16. Robinson, A., et al. 1996. Characterization of Grb2-binding proteins in human platelets activated by Fc gamma RIIA cross-linking. Blood. 88:522-530.

17. Yanaga, F., et al. 1995. Syk interacts with tyrosine phopshorylated proteins in human platelets activated by collagen and cross linking of the Fc $\gamma$-IIA receptor. Biochem. J. 311:471-478.

18. Blake, R.A., Schieven, G.L., and Watson, S.P. 1994. Collagen stimulates tyrosine phosphorylation of phospholipase $\mathrm{C} \gamma 2$ but not phospholipase $\mathrm{C} \gamma 1$ in human platelets. FEBS Lett. 353:212-216.

19. Poole, A., et al. 1997. The Fc receptor gamma-chain and the tyrosine kinase Syk are essential for activation of mouse platelets by collagen. EMBO J. 16:2333-2341.

20. Gibbins, J., et al. 1996. Tyrosine phosphorylation of the Fc receptor gammachain in collagen-stimulated platelets. J. Biol. Chem. 271:18095-18099.

21. Nieuwenhuis, H.K., Akkerman, J.W., Houdijk, W.P., and Sixma, J.J. 1985. Human blood platelets showing no response to collagen fail to express surface glycoprotein Ia. Nature. 318:470-472.

22. Santoro, S.A., Rajpara, S.M., Staatz, W.D., and Woods, V.L., Jr. 1988. Isolation and characterization of a platelet surface collagen binding complex related to VLA-2. Biochem. Biophys. Res. Commun. 153:217-223.

23. Moroi, M., Jung, S.M., Okuma, M., and Shinmyozu, K. 1989. A patient with platelets deficient in glycoprotein VI that lack both collageninduced aggregation and adhesion. J. Clin. Invest. 84:1440-1445.

24. Ryo, R., et al. 1992. Deficiency of P62, a putative collagen receptor, in platelets from a patient with defective collagen-induced platelet aggregation. Am. J. Hematol. 39:25-31.

25. Gibbins, J.M., Okuma, M., Farndale, R., Barnes, M., and Watson, S.P. 1997. Glycoprotein VI is the collagen receptor in platelets which underlies tyrosine phosphorylation of the Fc receptor gamma-chain. FEBS Lett. 413:255-259. 
26. Asselin, J., et al. 1997. A collagen-like peptide stimulates tyrosine phosphorylation of syk and phospholipase $\mathrm{C} \gamma 2$ in platelets independent of the integrin $\alpha 2 \beta 1$. Blood. 89:1235-1242.

27. Watson, S.P., and Gibbins, J. 1998. Collagen receptor signalling in platelets: extending the role of the ITAM. Immunol. Today. 19:260-264.

28. Fujii, C., et al. 1994. Involvement of protein-tyrosine kinase p72syk in collagen induced signal transduction in platelets. Eur. J. Biochem. 226:243-248.

29. Chan, A.C., Iwashima, M., Turck, C.W., and Weiss, A. 1992. ZAP-70: a 70 $\mathrm{kd}$ protein-tyrosine kinase that associates with the TCR zeta chain. Cell. 71:649-662.

30. Reth, M. 1989. Antigen receptor tail clue. Nature. 338:383-384.

31. Iwashima, M., Irving, B.A., van Oers, N.S., Chan, A.C., and Weiss, A. 1994. Sequential interactions of the TCR with two distinct cytoplasmic tyrosine kinases. Science. 263:1136-1139.

32. Tsuji, M., Ezumi, Y., Arai, M., and Takayama, H. 1997. A novel association of Fc receptor gamma-chain with glycoprotein VI and their co-expression as a collagen receptor in human platelets. J. Biol. Chem. 272:23528-23531.

33. Hung, D.T., Wong, Y.H., Vu, T.K., and Coughlin, S.R. 1992. The cloned platelet thrombin receptor couples to at least two distinct effectors to stimulate phosphoinositide hydrolysis and inhibit adenylyl cyclase. J. Biol. Chem. 267:20831-20834.

34. Offermanns, S., Laugwitz, K.L., Spicher, K., and Schultz, G. 1994. G proteins of the G12 family are activated via thromboxane A2 and thrombin receptors in human platelets. Proc. Natl. Acad. Sci. USA. 91:504-508.

35. Raab, M., da Silva, A.J., Findell, P.R., and Rudd, C.E. 1997. Regulation of Vav-SLP-7 6 binding by ZAP-70 and its relevance to TCR zeta/CD3 induction of interleukin-2. Immunity. 6:155-164.

36. Turner, M., et al. 1995. Perinatal lethality and blocked B-cell development in mice lacking the tyrosine kinase Syk. Nature. 378:298-302.

37. Cheng, A.M., et al. 1995. Syk tyrosine kinase required for mouse viability and B-cell development. Nature. 378:303-306.

38. Shivdasani, R.A., et al. 1995. Transcription factor NF-E2 is required for platelet formation independent of the actions of thrombopoietin/MGDF in megakaryocyte development. Cell. 81:695-704.

39. Offermanns, S., Toombs, C.F., Hu, Y.H., and Simon, M.I. 1997. Defective platelet activation in G alpha(q)-deficient mice. Nature. 389:183-186. 